\title{
INVESTIGATIONS OF AGNS BY THE INTERPLANETARY SCINTILLATION METHOD
}

\author{
VADIM S. ARTYUKH \\ Pushchino Radio Astronomy Observatory of the Lebedev Physical Institute RAS, Leninskii pr. 53, \\ 117924, Moscow, Russia
}

\begin{abstract}
This is a brief revicw of investigations of AGNs by the interplanetary scintillation method made in Pushchino Radio Astronomy Observatory.
\end{abstract}

Keywords: compact radio source, active galactic nucleus, physical conditions, cosmology, interplanetary scintillations

Since 1978 investigations of AGNs have been carried out at Pushchino Radio Astronomy Observatory (PRAO) by the interplanetary scintillation (IPS) method. All observations of compact radio sources located in the AGNs were made with the Large Phased Array (LPA) at a frequency of $102 \mathrm{MHz}$. The sensitivity of the observations was $\sim 0.1$ Jy owing to the LPA being the largest radio telescope in the meter radio wave range $\left(\right.$ Ageom $\left.=70000 \mathrm{~m}^{2}\right)$.

\section{IPS Method}

The diffraction of radio waves by inhomogeneities of the interplanetary plasma evokes an inhomogeneous distribution of radiation intensity of radio source emission at the Earth's surface. Because the interplanetary plasma moves away from the Sun we see fluctuations of the intensity with time (Hewish et al., 1964).

A power spectrum of the IPS depends on both the interplanetary plasma spectrum and the brightness distribution of the radio source. Shishov and Shishova (1978) have calculated theoretical spectra for radio sources with different angular diameters. Comparing the observed spectrum with theoretical spectra we determine the angular diameter of the scintillating radio source.

To exclude fluctuation of the solar wind velocity we take the mean spectrum for 7 - 10 days of observations. The dependence of the mean solar wind velocity on the solar activity and on the helio-latitude was taken into account.

The resolution of the IPS method depends on the signal to noise ratio. The limiting resolution of the method is determined by the source noise in the case of extremely strong sources, when the signal to noise ratio is greatest. At $102 \mathrm{MHz}$ the limit resolution is $\sim 0.01^{\prime \prime}$ (Artyukh and Shishova, 1987). An interferometer with that resolution at $102 \mathrm{MHz}$ would have a baseline of $60000 \mathrm{~km}$.

The IPS method is especially effective in the meter wave rang where no VLBI systems work now. 


\section{Interstellar Scattering}

The interstellar scattering of the radio emission, caused by galaxies along the line of sight from the radio source to the observer and by the intergalactic medium, increases the apparent angular size of the compact radio source. The scattering angle is $\theta \sim v^{-2} L^{1 / 2}$ (Chandrasekhar, 1952), where $v-$ the frequency and L the thickness of the scattering layer. When $\mathrm{L}$ approaches infinity $\theta$ must approach infinity too. This means that we cannot see compact radio sources in very distant AGNs. But this is not correct. Due to the redshift the effective scattering takes place in the layers (galaxies) located near our Galaxy with redshift $\mathrm{z}<0.4$ (Artyukh, 1997). More distant galaxies scatter the radiation at higher frequencies and we can ignore this scattering.

\section{Results of the Investigations of AGNs}

The investigations of AGNs were made in two directions:(1) - investigations of physical conditions in AGNs and (2) - cosmology.

\subsection{Physical CONDItions in AGNS}

1. Many compact radio sources located in AGNs have low frequency cutoffs in their spectra. An analysis of physical mechanisms responsible for the spectral cutoffs showed that synchrotron self-absorption of the radiation is the most likely mechanism for many radio sources. In that case we can estimate magnetic field strengths, relativistic electron number densities, and corresponding magnetic field and relativistic plasma energies in the AGNs where these radio sources are located (Artyukh, 1990).

The results were published, among others, in (Artyukh and Oganissian, 1989; Artyukh et al., 1999; Tyul'bashev and Chernikov, 2000). Typical magnetic field strengths are $10^{-3}-10^{-4} \mathrm{~g}$ at the scale $\sim 10^{2} \mathrm{pc}$. Energy equipartition is not in effect in many AGNs in spite of the roughness of the estimates.

There are 3 radio sources CTA 21, CTA 102 and 4C 34.07 where estimates of the magnetic field strengths are unreasonably low, $\sim 10^{-8} \mathrm{G}$ (Artyukh et al., 1999). This is probably due to the Doppler boosting effect. When a radio source moves towards an observer with a relativistic velocity the observer registers a flux density $S$ larger than the flux density of the motionless radio source. Because the magnetic field strength estimate $\mathrm{H} \sim \mathrm{S}^{-2}$ (Artyukh, 1990) the estimated $\mathrm{H}$ is less than the real value.

The procedure for estimating the physical parameters in Artyukh (1990) is based on the model of a homogeneous radio source. We use this procedure when information about the spectra of radio sources is very poor. If we have the detailed shape of the low frequency cutoff of the spectrum we obtain information about the 
magnetic field and relativistic plasma distribution in the radio source (Artyukh and Chernikov, 2000).

2. A correlation was found between physical conditions in AGNs and the morphological types of their host galaxies. Magnetic field strengths are smaller in the AGNs in spiral galaxies (accordingly the relativistic electron number densities are higher) than in the AGNs in elliptical galaxies (Artyukh, 1987).

3. Observations of 5 giant radio galaxies (3C 236, 3C 219, DA 240, NGC 315, NGC 1275)(Artyukh and Ogannisian, 1988(a); 1993; Artyukh et al., 1994) point to the possible relationship between radio structures of the radio galaxies and physical conditions in the nuclei of their parent galaxies. For radio galaxies of classical morphology (where giant radio clouds are connected by thin jets with the nucleus) the energy of the magnetic field in the nuclei considerably exceeds the energy of relativistic electrons. The opposite is true for jetless radio galaxies; for radio tailed galaxies an approximate equipartition is observed.

We will continue the investigations of giant radio galaxies to obtain a reliable statistical result.

\subsection{Cosmology}

1. A survey of compact (scintillating) radio sources was made with the LPA at 102 MHz. The survey covered 2 areas of the $7 \mathrm{C}$ survey with total area 0.144 ster. We have detected 395 compact radio sources, of which 380 were found for the first time (Artyukh and Tyul'bashev, 1996; Artyukh et al., 1998).

The scintillating source count showed a sharp decrease at weak flux densities compared to that for extended radio sources. We have analysed all known origins for the drop of the compact radio source count: an absorption and a scattering of the radio emission, measurement errors, the confusion of scintillating radio sources, gravitational lensing, geometrical effects and cosmological evolution. Most probably this effect is a result of a high birth rate of quasars compared with the birth rate of radio galaxies during the course of their cosmological evolution (Artyukh and Tyul'bashev, 1998).

2. Observing scintillating radio sources in different areas we always analyse the distribution of the sources on the sky surface. Everywhere we saw a homogeneous distribution of scintillating radio sources (Artyukh, 2000). But in the direction to the radio galaxy DA 240 we have found a big deficit of compact radio sources. The angular scale of the 'radio void' is $\sim 5^{0}$ and the linear scale is $\sim 10^{3} \mathrm{Mpc}$ (Artyukh and Ogannisian,(b) 1988). It compares with the dimension of the Universe.

\section{Acknowledgements}

This work was partly supported by the grant RFBR \#00-02-17845. The author's participation in IAU Colloquium 182 was supported by the China Local Organizing 
Committee (Chairperson, Prof. Rendong Nan). I thank Prof. R. Strom for the help in the preparation of this paper.

\section{References}

Artyukh, V.S.: 1987, The Radio Spectra of Scintillating Sources, and the Morphological Type of the Active Host galaxies, Soviet Astronomy Letters 13, 108-112.

Artyukh, V.S.: 1990, Interplanetary Scintillation Observations of Meter-Wavelength Radio Emission from Galaxies, in: N.G. Basov (ed.), Proceedings of the Lebedev Physics Institute, Academy of Sciences of the USSR, 189, pp. 289-309. Nova Science Publishers New York/Budapest.

Artyukh, V.S.: 1997, The Influence of Scattering of Radio Emission on Scintillating Radio Source Counts, Astron. Rep. 41, 160-162.

Artyukh, V.S.: 2000, A Search of Large-Scale Inhomogeneities in a Compact Radio Source Distribution, Astron. Rep. 44, 349-352.

Artyukh, V.S. and Chernikov, P.A.: 2001, Synchrotron Spectra of Inhomogeneous Radio Sources, Astron. Rep. 45, 16-25.

Artyukh, V.S. and Ogannisian, M.A.: 1988(a), The Observation of the Giant Radio Galaxies 3C 236 and DA 240 at $102 \mathrm{MHz}$, Pis'ma v Astronomy Journal 14, 706-712.

Artyukh, V.S. and Ogannisian, M.A.: 1988(b), A Large-Scale Void in the Distribution of Compact Radio Sources, Soviet Astronomy Letters 14, 377-378.

Artyukh, V.S. and Ogannisian, M.A.: 1993, The Investigation of the Perceus Cluster of Galaxies at $102 \mathrm{MHz}$, Astronomicheskii J. 70, 443-450.

Artyukh, V.S., Ogannisian, M.A. and Tyul'bashev, S.A.: 1994, Observations of the Radio Galaxies NGC 315 and 3C 219 at $102 \mathrm{MHz}$, Pis'ma v Astron. J. 20, 178-183.

Artyukh, V.S. and Shishova, T.D.: 1987, About the Resolution of the IPS Method, Radiofisika 30, 1396.

Artyukh, V.S. and Tyul'bashev, S.A.: 1996, The Cosmological Evolution of Compact Radio Sources from $102 \mathrm{MHz}$ Observations, Astron. Rep. 40, 661-668.

Artyukh, V.S. and Tyul'bashev, S.A. 1998, The Cosmological Evolution of Quasars with Steep Spectra, Astron. Rep. 42, 576-586.

Artyukh, V.S., Tyul'bashev, S.A. and Chernikov, P.A.: 1999, Investigations of Compact SteepSpectrum Radio Sources Using $102 \mathrm{MHz}$ Interplanetary Scintillation Observations, Astron. Rep. $43,1-12$.

Artyukh, V.S., Tyul'bashev, S.A. and Isaev, E.A.: 1998, The Cosmological Evolution of Compact Radio Sources from $102 \mathrm{MHz}$ Observations, Astron. Rep. 42, 283-292.

Chandrasekhar, S.: 1952, A Statistical Basis for the Theory of Stellar Scintillation, MNRAS 112. 475-484.

Hewish, A., Scott, P.F. and Wills, D.: 1964, Interplanetary Scintillation of Small Diameter Radio Sources, Nature 203, 1214-1217.

Shishov, V.I. and Shishova, T.D.: 1978, Influence of Source Sizes on the Spectra of Interplanetary Scintillations, Soviet Astron. 22, 235-239.

Tyul'bashev, S.A. and Chernikov, P.A.: 2000, Physical Conditions in Steep Spectrum Compact Radio Sources, Astron. Rep. 44, 286-299. 